\title{
Uma avaliação experimental do tubo de ondas sonoras estacionárias
}

\author{
An experimental evaluation of standing sound waves in pipes \\ Daniel Cosmo Pizetta ${ }^{1}$, Adilson Barros Wanderley $^{1}$, Valmor Roberto Mastelaro ${ }^{1}$, Fernando \\ Fernandes Paiva*1 \\ ${ }^{1}$ Instituto de Física de São Carlos, Universidade de São Paulo, São Carlos, SP, Brasil
}

Recebido em 9 de Novembro de 2016. Revisado em 20 de Dezembro de 2016. Aceito em 20 de Dezembro de 2016

A maioria dos aparatos experimentais para o estudo de ondas de som estacionárias é composto por um tubo onde há um êmbolo com um microfone para captação do sinal e um alto-falante como fonte excitadora. Este mesmo êmbolo define o tamanho útil do tubo de forma manual. A interpretação correta dos fenômenos físicos relacionados a este experimento está intimamente conectada com a configuração experimental, podendo causar má interpretação. O sistema usual foi adaptado para ser automatizado e para que permitisse a movimentação do êmbolo e do microfone de forma independente. Os resultados obtidos na configuração de tubo semiaberto e fechado estão em acordo com a teoria e mostraram quais parâmetros afetavam de forma mais significativa os experimentos em relação à montagem tradicional, como a falta de vedação entre o alto-falante e o tubo e o perfil do sinal que não corresponde ao de uma onda estacionária quando o microfone está fixo ao êmbolo. Além de permitir uma correta interpretação do fenômeno, a utilização de um sistema automatizado para aquisição do sinal permitiu a observação de resultados experimentais que normalmente não são discutidos quando este tipo de experimento é realizado.

Palavras-chave: tubo de ondas sonoras estacionárias, ondas longitudinais, modos normais, laboratório de física, problemas experimentais.

Typical experimental settings for the study of standing sound waves include a pipe, a microphone attached to a piston for signal acquisition and a speaker connected to a generator for the production of waves. The piston can be used for manual adjustments in the effective size of the tube. The correct interpretation of physical phenomena related to such an experiment is closely connected to the experimental setup, as the speaker position relative to the pipe and the microphone movement relative to the piston. The usual system was automated and adapted for enabling the independent movement of both piston and microphone and sound waves were studied in semi-open and closed pipe configurations. The results are in agreement with the theory and showed the parameters that mostly affected the experiments in comparison to the traditional setup. Two of such parameters are lack of sealing between the speaker and the pipe, and signal profile, which does not correspond to a standing wave when the microphone and the piston are attached to each other. The use of an automated system for signal acquisition enables the observation of experimental results that are not usually discussed and the correct interpretation of the standing wave phenomenon.

Keywords: standing sound wave pipe, longitudinal waves, normal modes, physics laboratory, experimental problems.

*Endereço de correspondência: paiva@ifsc.usp.br 


\section{Introdução}

O conceito de ondas, em especial de ondas estacionárias, está presente em quase todos os currículos dos cursos de ciências exatas, como Física, seja em cursos de ensino superior ou no ensino médio $[1$, 2]. Existem várias maneiras de se visualizar ondas em estado estacionário, sendo a mais simples delas o experimento da corda vibrante baseado no conceito de ondas transversais $[3,4]$. No caso de ondas longitudinais, as ondas de som são as mais comumente utilizadas e é, neste caso, que se encontram as dificuldades na visualização e interpretação correta dos fenômenos envolvidos. O fato das ondas longitudinais oscilarem na mesma direção de propagação representa um maior grau de dificuldade em relação a sua interpretação gráfica e, como consequência, a sua compreensão [3, 4]. Além disso, é necessário um cuidado ainda maior na interpretação teórica neste caso, que muitas vezes negligencia fatores experimentais que são cruciais para o correto entendimento do fenômeno, tais como a distância da fonte sonora, quais casos são considerados - tubos abertos ou fechados - as frequências de resposta dos elementos do sistema, etc. [5-8]. Comumente, a montagem experimental de ondas sonoras estacionárias utiliza um tubo contendo um êmbolo para a variação do comprimento útil do tubo, um gerador de áudio acoplado a um alto-falante e um dispositivo para aquisição do sinal (microfone), como apresentado na Figura $1[1,2,9]$.

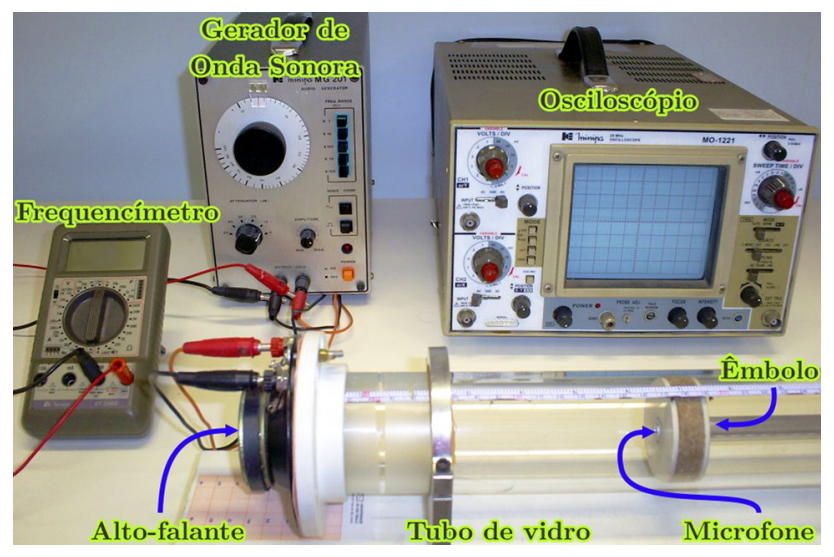

Figura 1: Equipamento básico para a prática de ondas e oscilações. O aparato é formado por um tubo transparente com um pistão móvel no qual se encontra um microfone fixo à extremidade, um gerador de onda ligado a um alto-falante e a um frequencímetro e um voltímetro ou um osciloscópio para visualização do sinal
Na maioria dos materiais didáticos descritos na literatura, bem como dos produtos comerciais destinados ao ensino de física, o êmbolo e o microfone são um conjunto único, isto é, o movimento do êmbolo está condicionado ao movimento do microfone e vice-versa. Esta limitação prática exige alguns cuidados ao se avaliar o que ocorre entre dois pontos consecutivos de máximos no sinal adquirido, podendo surgir dúvidas sobre a formação ou não de ondas estacionárias entre estes pontos. Afinal, se o comprimento do tubo está variando e a frequência está fixa, o que estamos medindo? Outro fator prejudicial neste tipo de montagem está relacionado à medida ou forma de obtenção da amplitude do sinal sonoro, que normalmente é lida por meio de um voltímetro ligado ao microfone ou através da tela de um osciloscópio. A utilização do voltímetro faz com que sejam detectados somente os pontos de máximos não permitindo obter uma ilustração durante a trajetória do êmbolo. Por outro lado, a utilização do osciloscópio pode causar confusão em relação ao que se observa na tela e o que está ocorrendo fisicamente no interior do tubo, ou seja, o aluno pode se perguntar se o que está vendo no osciloscópio é ou não a onda estacionária que está sendo medida.

Tendo em vista estes aspectos, realizou-se um estudo sobre ondas estacionárias em um tubo com a finalidade de sanar os principais problemas experimentais e sua interpretação teórica de forma complementar ao estudado por Vieira [2]. Além disso, está sendo proposta uma forma diferente para a aquisição do sinal, a qual evitará o surgimento de dúvidas ou dubiedade nos fenômenos observados, incluindo exemplos e forma de analisá-los. Somado a isto, serão discutidos procedimentos afim de evitar a interferência dos sinais (no caso de mais de um dispositivo em sala de aula), bem como dicas sobre como construir um equipamento pequeno e de baixo custo.

\section{Aspectos Teóricos}

Considerando o som como uma onda mecânica, podemos definir sua velocidade, $v$, como sendo a distância entre a formação de dois máximos consecutivos de amplitude, de comprimento de onda $\lambda$, dividida pelo período $T$ em que este fenômeno ocorre. Esse período é definido como o inverso de sua frequência $f$. Com isso, temos na equação (1) a expressão para 
a velocidade de propagação da onda sonora $[3,4,8$, 10].

$$
v=\lambda f
$$

O estudo de ondas estacionárias pode ser considerado simples se supusermos a propagação de ondas planas. Essa suposição permite desconsiderar as reflexões na superfície lateral do tubo, fazendo com que nosso sistema possa ser tratado como unidimensional. A condição necessária para que isso seja válido é que a frequência do gerador de sinais seja mantida abaixo da chamada frequência de corte $f_{c}$. Para um tubo cilíndrico de diâmetro $D$, onde a velocidade do som é dada por $v$, podemos utilizar a equação (2) para o cálculo da frequência de corte. Esta equação pode ser deduzida a partir das condições de contorno da borda lateral do tubo em um tratamento bidimensional $[8,10]$.

$$
f_{c}=\frac{1,84 v}{\pi D}
$$

A formação de ondas estacionárias ocorre, por exemplo, no encontro entre duas ondas com características similares (mesmas amplitude e velocidade, porém, fases opostas). Como as oscilações para ondas longitudinais estão na mesma direção do deslocamento, sua representação gráfica já apresenta um primeiro desafio, como exemplificado na Figura 2.a. Os vetores azuis indicam a amplitude e a direção de deslocamento para as partículas situadas em sua base. Para simplificar o perfil da onda podemos, então, representar as amplitudes de forma perpendicular ao deslocamento como indicado pelas setas vermelhas, fazendo com que o perfil seja mais facilmente visualizado. Na Figura 2.b, já utilizando o perfil de forma perpendicular para a amplitude de deslocamento, inserimos também amplitude da pressão (linhas pretas contínuas) e a representação das moléculas do gás no interior do tubo (círculos azuis). É importante observar que o deslocamento mostrado pelas setas azuis no item $a$ da Figura 2 representa a distribuição das moléculas no item $b$. Desta forma, no local onde os vetores têm amplitude maior, o gás está rarefeito, indicando uma expansão. Em contraste, onde o vetor é menor, o gás está mais denso, representando uma compressão. Assim, o máximo de pressão corresponde ao mínimo de deslocamento e vice-versa.

Outro fato a ser observado é que nas extremidades de um tubo fechado não deverá haver deslocamento, uma vez que existe uma parede que impede tal

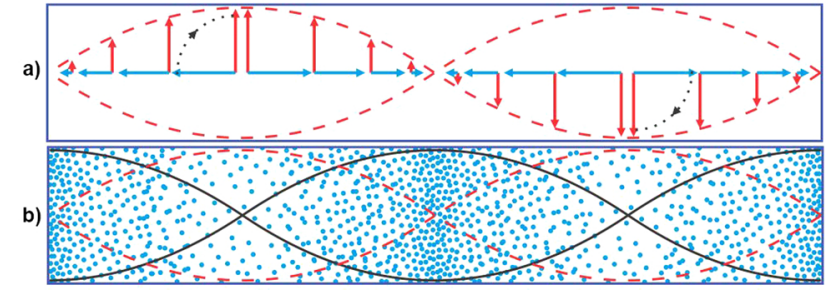

Figura 2: Ilustração dos modos normais dentro de um tubo fechado. a) Representação do deslocamento das partículas através dos vetores azuis. Vetores e linha tracejada vermelha representam o mesmo deslocamento na perpendicular. b) A linha preta contínua representa a amplitude de pressão do gás, a linha vermelha tracejada indica a amplitude de deslocamento das moléculas e o pontilhado indica a distribuição de moléculas do interior do tubo.

movimento. Neste ponto existirá, então, um mínimo de deslocamento o que corresponde a um máximo de pressão.

\subsection{Tubo fechado}

Utilizando a análise dos máximos e mínimos de pressão, ilustrados na Figura 2.b, podemos obter os modos normais de uma onda longitudinal relacionando o comprimento do tubo $L$ com o comprimento de onda $\lambda$ para o tubo fechado (indicado pelo índice $f$ ), através da equação (3) [4].

$$
f_{f}=n_{f} \frac{v}{2 L} \quad \text { onde } \quad n_{f}=1,2,3 \ldots
$$

Nesta configuração, os possíveis $n$ podem ser quaisquer números inteiros, pares ou ímpares, maiores que zero. Os perfis nos três primeiros modos normais, $m$, estão representados na Figura 3.

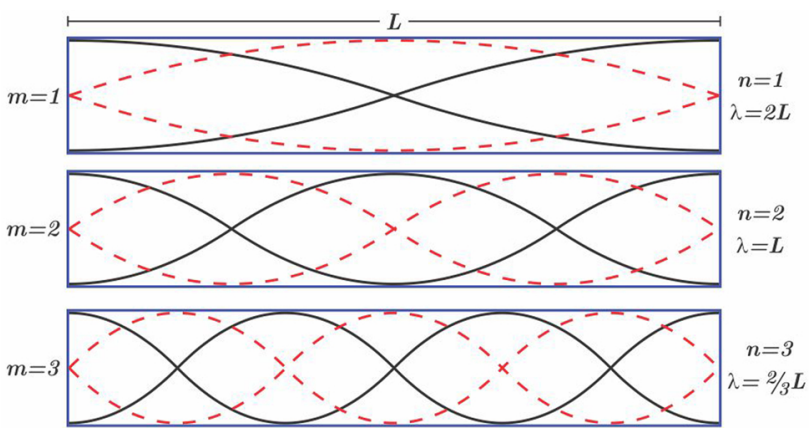

Figura 3: llustração dos três primeiros modos normais $(m)$ dentro de um tubo fechado de comprimento fixo, $L$, com indicação do comprimento $(\lambda)$ e número $(n)$ de onda. A linha preta contínua indica a amplitude da pressão no interior do tubo enquanto a linha vermelha tracejada indica a amplitude de deslocamento das moléculas. 


\subsection{Tubo semiaberto}

De maneira análoga, podemos analisar um tubo aberto em apenas uma extremidade, nomeado aqui como semiaberto e indicado pelo índice $s$, em que as frequências dos modos normais são obtidas de acordo com a equação (4) e ilustrados na Figura 4 [4], desconsiderando a aproximação terminal [11].

$$
f_{s}=n_{s} \frac{v}{4 L} \quad \text { onde } \quad n_{s}=1,3,5 \ldots
$$

Note que, neste caso, os possíveis valores de $n$ são somente os inteiros ímpares e que, de forma análoga ao anterior, não existem valores menores que 1 .

É importante ressaltar que $n$ representa um número que satisfaz as equações e as condições de contorno (também chamado de número de onda) e está relacionado à nomenclatura dos modos normais, $m$, de forma ordinária. Por exemplo, para o tubo fechado o primeiro modo normal $(m=1)$ é representado por $n=1$, já o terceiro modo normal $(m=3)$ por $n=3$. Para o tubo semiaberto o primeiro modo normal é representado por $n=1$, como o anterior, porém, o terceiro modo normal é representado por $n=5$. Assim temos a expressão para o $m$-ésimo modo normal em relação ao número de onda $n$ para o tubo fechado (equação (5p) e semiaberto (equação (6)).

$$
\begin{gathered}
m_{f}=n_{f} \\
m_{s}=\frac{n_{s}+1}{2}
\end{gathered}
$$

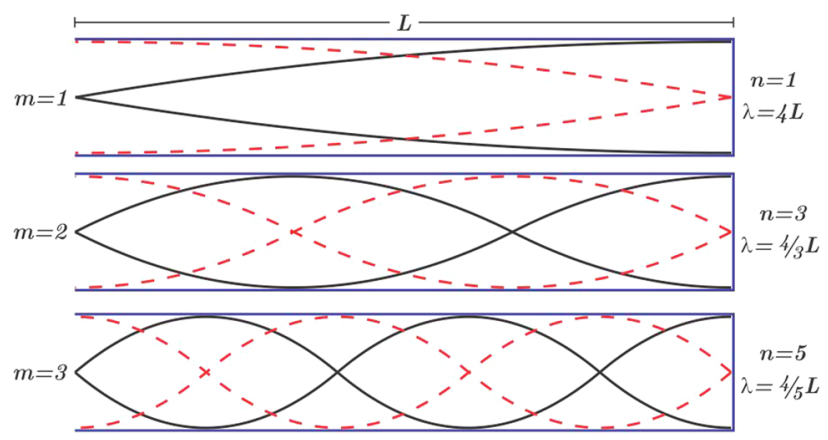

Figura 4: Ilustração dos três primeiros modos normais $(m)$ dentro de um tubo semiaberto (extremidade esquerda aberta) de comprimento fixo, $L$, com indicação do comprimento $(\lambda)$ e número $(n)$ de onda. A linha preta contínua indica a amplitude da pressão no interior do tubo enquanto a linha vermelha tracejada indica a amplitude de deslocamento das moléculas.

\section{Materiais e Métodos}

O sistema utilizado para o experimento é composto por quatro partes, como apresentado pelo diagrama esquemático da Figura 5.

A primeira parte do aparato é formada por um tubo de material transparente (vidro) que possui um êmbolo que permite ajustar seu comprimento útil interno. O tubo possui um diâmetro interno de 53 $\mathrm{mm}$ e espessura de $3 \mathrm{~mm}$. Coaxial ao êmbolo existe uma vareta onde é fixado o microfone. Isso permite que o êmbolo e o microfone possam se movimentar independentemente ou em conjunto. Compondo a segunda parte temos um alto-falante que é posicionado na extremidade do tubo, conectado a um gerador de onda e um frequencímetro. O alto-falante do tipo twitter (que possui uma melhor resposta para altas frequências, acima de $1 \mathrm{kHz}$ ) pode, segundo um mecanismo de suporte, ser fixado à extremidade do tubo ou permanecer afastado dela. Na terceira parte, o conjunto formado pelo êmbolo e pela vareta pode ser acoplado a um motor de passo através de uma correia dentada que fará, programaticamente, sua movimentação. O motor está ligado ao driver L298N e este, por sua vez, ao Arduino Uno v3 e a uma fonte de tensão contínua que fornece corrente para a alimentação do motor. O programa gravado no Arduino se comunica através da porta serial (USB) com um programa escrito em Python no computador. Finalmente, na quarta parte, o mi-

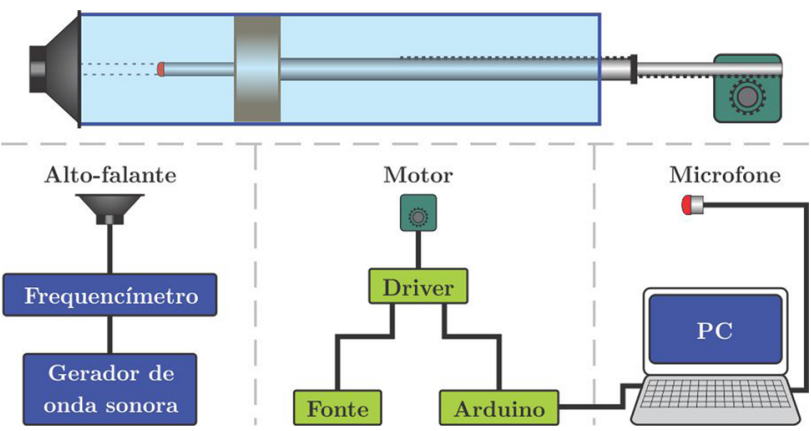

Figura 5: Diagrama esquemático do sistema eletromecânico para medida de ondas sonoras no interior do tubo. O sistema é dividido em quatro partes. a) Tubo com êmbolo e vareta para microfone - que se movimentam independentemente. b) Gerador de onda, frequencímetro e alto-falante. c) Arduino, driver para controle do motor em conjunto com uma fonte de tensão contínua e motor de passo. d) Microfone de eletreto ligado ao computador para aquisição do sinal e controle do motor. 
crofone de eletreto, também ligado ao computador, possibilita a captura do sinal (Figura 6).

O programa em Python faz a gravação do sinal de áudio no formato padrão de som (WAV), além de controlar a movimentação do motor de passo através do controle do Arduino. Os programas utilizados podem ser acessados no repositório público do Github (https://github.com/ dpizetta/stading-sound-waves). E importante mencionar que toda a aquisição do sinal de áudio foi realizada com o motor parado, devido a inserção de ruído quando o mesmo está em movimento. Desta forma, a comunicação se faz necessária para que a gravação e a movimentação sejam realizadas em momentos distintos, descritos pelo fluxograma do algoritmo utilizado, Figura 7 [12].
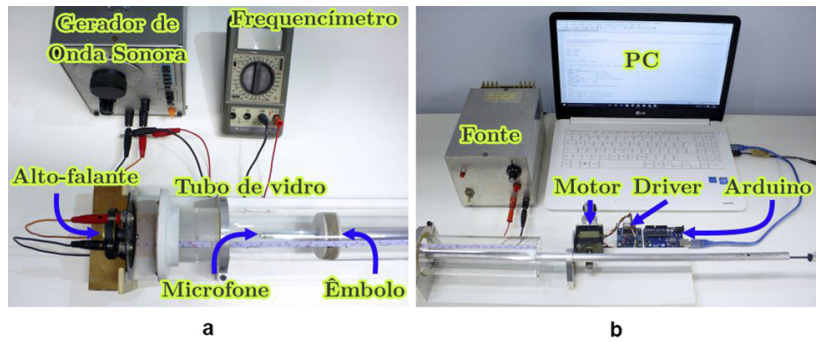

Figura 6: Foto da montagem experimental utilizada para aquisição de dados. a) Gerador de sinal e frequencímetro ligado ao alto falante, evidenciando também o tubo que contém o êmbolo e o microfone para aquisição com movimentação separada. b) Extremidade oposta do tubo onde o motor de passo faz o movimento do êmbolo ou microfone. $\mathrm{O}$ motor está ligado ao driver alimentado pela fonte e também ao Arduino controlado pelo computador.

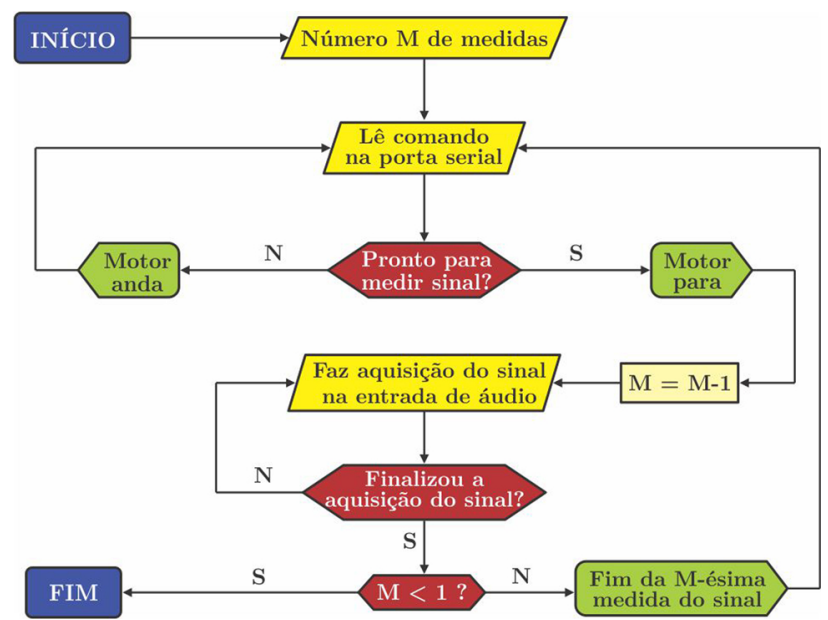

Figura 7: Fluxograma do algoritmo para aquisição do sinal e controle do motor de passo englobando o programa do computador (em Python) em conjunto com o programa do Arduino (em $\mathrm{C}++)$.
A taxa de amostragem na aquisição do sinal foi de $20 \mathrm{kHz}$ e o tempo de gravação de cada passo foi de $0,5 \mathrm{~s}$. Essa taxa de amostragem é cerca de 3 a 4 vezes maior do que das frequências do gerador de onda sonora de aproximadamente $3 \mathrm{kHz}$. O movimento do êmbolo ou do microfone por meio do motor foi realizado com um passo de $2,5 \mathrm{~mm}$. O microfone inicia seu movimento sempre da superfície do alto-falante, não encostando no mesmo. $\mathrm{O}$ erro no posicionamento dos objetos é de $5 \mathrm{~mm}$, tendo sido desconsiderado uma vez que estava presente em todos os experimentos que serão comparados.

Em todos os experimentos a temperatura ambiente foi mantida em $T=(23 \pm 1)^{\circ} \mathrm{C}$ através do uso do ar-condicionado. Além disso, foi considerada a pressão como $1 \mathrm{~atm}$ e a umidade relativa do ar de $50 \%$. Com isso, a velocidade do som no ar é dada por $v=(345 \pm 3) \mathrm{m} / \mathrm{s}$. A flutuação foi obtida considerando variações de umidade em $25 \%$ e pressão em 0,001 atm [6]. A seguir descrevemos os detalhes de cada experimento realizado neste trabalho.

\subsection{Tubo fechado com comprimento fixo}

Para o experimento do tubo fechado com comprimento fixo, foi acoplado um anel de borracha que garante a vedação do alto-falante ao tubo. Este é um item importante neste experimento pois, como mostraremos mais adiante nos resultados do experimento do tubo semiaberto, apenas $1 \mathrm{~mm}$ de deslocamento do alto-falante faz com que, naquela extremidade, o tubo possa ser considerado aberto. Nesta configuração, $l$ representa a posição do microfone e $L$ o comprimento fixo do tubo, determinado pela posição do êmbolo. Observe na Figura 8 que a extremidade esquerda é fechada com o próprio alto-falante e vedada na região de contato deste com o tubo. O microfone se move a partir da extremidade esquerda (plano definido pela superfície do alto-falante) e segue até a superfície do êmbolo mantendo o comprimento útil do tubo fixo (êmbolo fixo).

Nesta configuração, foi utilizada uma frequência de excitação $\left(f_{e}=3300 \mathrm{~Hz}\right)$ para dois comprimentos de tubo $\left(L_{1}=10,5 \mathrm{~cm}\right.$ e $\left.L_{2}=26,0 \mathrm{~cm}\right)$ distintos. Foi, ainda, realizado um experimento utilizando uma frequência de excitação $\left(f_{e}=3150 \mathrm{~Hz}\right)$ fora da frequência do modo normal para um comprimento de tubo determinado $(L=15,0 \mathrm{~cm})$ afim de se verificar, ou não, a formação de ondas estacionárias. 


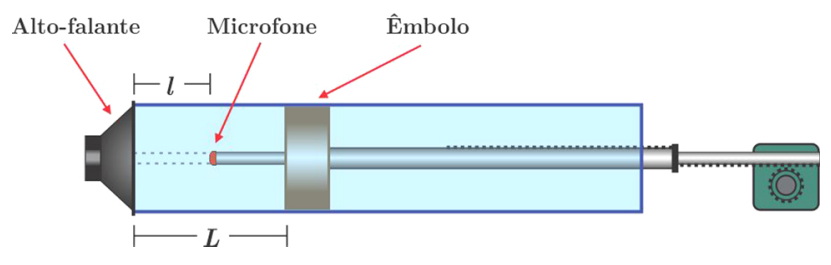

Figura 8: Diagrama do experimento para o tubo fechado. A extremidade esquerda é fechada com o próprio alto-falante e a vedação garantida por um anel de borracha. O microfone inicia seu movimento da extremidade esquerda (plano definido pela superfície do alto-falante) e segue até a superfície do êmbolo, mantendo o comprimento $L$ fixo.

\subsection{Tubo semiaberto de comprimento fixo}

Para o experimento com o tubo semiaberto (apenas uma extremidade aberta), além dos parâmetros já descritos no item anterior, temos a distância do alto-falante à extremidade do tubo, denominada $d$, indicada na Figura 9.

A distância entre o alto-falante e a extremidade do tubo foi variada manualmente para cada medida ( $d$ entre $0,1 \mathrm{~cm}$ e $4,5 \mathrm{~cm}$ ) mantendo o comprimento do tubo fixo $(L=13,0 \mathrm{~cm})$. Com os dados adquiridos foram estudados os efeitos de cada um dos parâmetros no perfil da onda adquirida. Deste modo, pôde-se determinar a melhor configuração para esse sistema semiaberto, e essa configuração foi utilizada para o estudo utilizando, de maneira análoga ao experimento anterior, duas frequências distintas $\left(f_{1}=1200 \mathrm{~Hz}\right.$ e $\left.f_{2}=3300 \mathrm{~Hz}\right)$, para um comprimento fixo de tubo $(L=23,5 \mathrm{~cm})$ e posição fixa do alto-falante $(d=-4,5 \mathrm{~cm})$.

\subsection{Tubo fechado com comprimento variando}

Para o caso do tubo fechado variando o comprimento, $L$ representa, agora, o comprimento do tubo

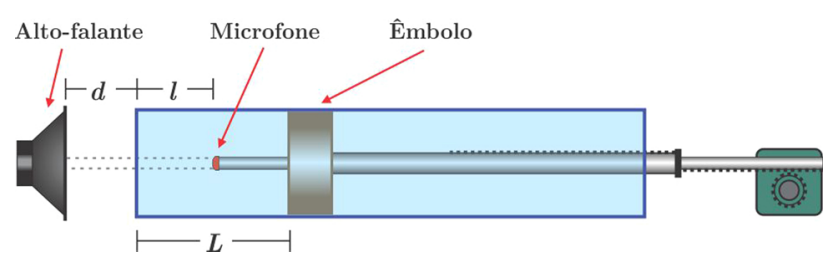

Figura 9: Diagrama do experimento para o tubo semiaberto (uma extremidade aberta - esquerda). A extremidade aberta mantém uma distância $d$ do alto-falante, que pode ser variada manualmente. Observe que o microfone sempre parte do plano definido pela superfície do alto-falante e adentra no tubo até a superfície do êmbolo. e também a posição do microfone, que permanece fixo em relação a extremidade do êmbolo $(L=l)$, como pode ser observado na Figura 10.

Esta montagem é similar a dos equipamentos comumente utilizados nos laboratórios de ensino (incluindo os equipamentos comerciais) e que será de extrema importância nas discussões dos problemas que este artigo enfatiza sobre ondas estacionárias no tubo. Neste caso, foram utilizadas duas frequências distintas $\left(f_{1}=1200 \mathrm{~Hz}\right.$ e $\left.f_{2}=3300 \mathrm{~Hz}\right)$ para um comprimento de tubo $L$ variando entre $0,0 \mathrm{~cm}$ e 25,0 cm. Refletiremos sobre as questões expostas na introdução, de interesse prático para a detecção de possíveis falhas e problemas que podem ocorrer nos equipamentos e na interpretação dos dados.

\subsection{Processamento dos dados}

Todos os dados obtidos foram processados utilizando o software OriginLab®, seguindo os procedimentos descritos a seguir:

- Extração do envelope do sinal original utilizando a função Envelope, que cria dois conjuntos de dados (partes superior e inferior).

- Suavização com filtro FFT ( Fast Fourier Transform) com 25000 pontos.

- Conversão da escala temporal para escala métrica dada pelo tempo de aquisição e comprimentos utilizados.

- Normalização do eixo das ordenadas com base na curva com maior amplitude para cada conjunto de medidas.

\section{Resultados e discussão}

A Figura 11 mostra um dado representativo do arquivo de áudio adquirido em que foram realizados

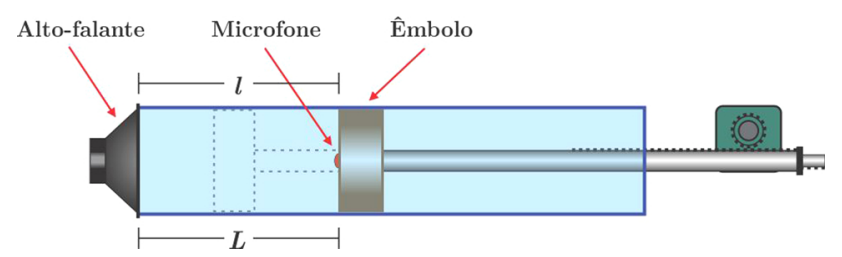

Figura 10: Diagrama do experimento para o tubo fechado com variação no comprimento $L$. Neste caso o comprimento do tubo é variado pela atuação do motor sobre o êmbolo. Note que neste caso, o microfone fica estacionário na extremidade do êmbolo, movendo-se em conjunto com o mesmo $(L=l)$. 


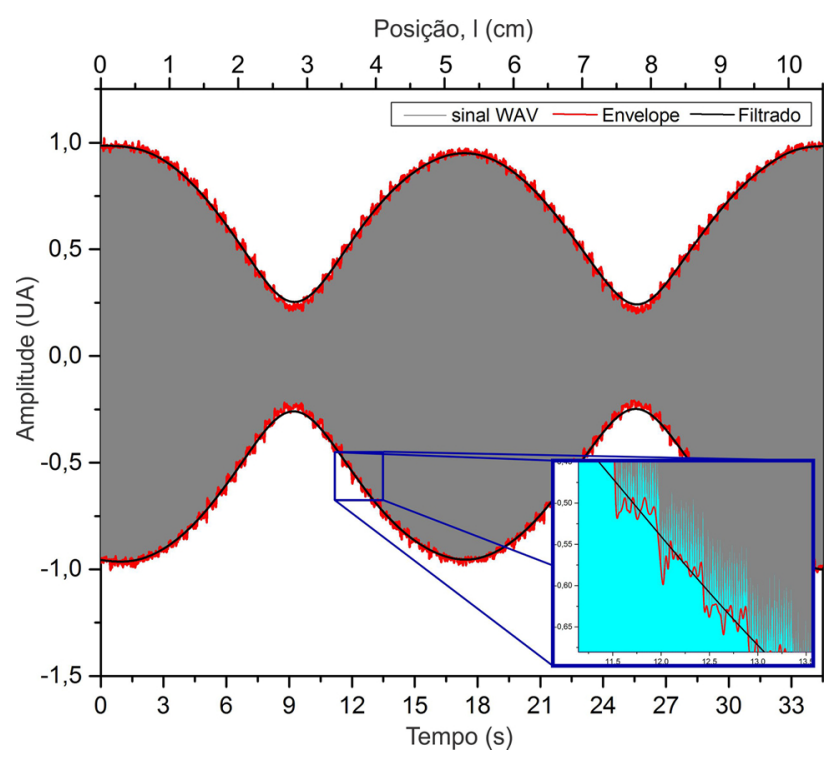

Figura 11: Dado bruto representativo mostrando as diferentes características do sinal até a obtenção do envelope da onda filtrada. Na ampliação, pode-se notar que os valores medidos são praticamente constantes durante cada aquisição (passo de 0,5 s).

os procedimentos descritos anteriormente para se extrair o envelope da onda. A escala de tempo foi convertida para cada medida de acordo com os valores de passo e tamanho do tubo configurados a cada etapa. Em todos os outros gráficos que seguem serão mostradas apenas as curvas do envelope já filtrado, para facilitar a visualização. Os dados brutos bem como demais dados extras podem ser acessados nos materiais suplementares.

\subsection{Tubo fechado com comprimento fixo - frequência do modo normal}

Na Figura 12 apresentamos os resultados de um experimento simples que representa com grande exatidão o que a teoria nos diz, como dado pelas equações (3) e (5). Neste caso, foram medidos os sinais para dois comprimentos distintos $\left(L_{1}=10,5\right.$ $\mathrm{cm}$ e $L_{2}=26,0 \mathrm{~cm}$ ), dada uma mesma frequência de excitação de $f_{e}=3300 \mathrm{~Hz}$. A curva tracejada representa a curva teórica.

Conforme esperado, o padrão obtido corresponde ao esperado de acordo com a teoria, com máximos de amplitude encontrado em posições compatíveis com a frequência utilizada. A pequena modificação na amplitude do sinal adquirido se dá pela perda (dissipação) causada pelo aumento na extensão do meio. Este experimento pode ser recomendado para o uso em sala de aula, devido à compatibilidade dos resultados com a teoria, sem a inserção de dubiedades ou problemas experimentais que possam confundir o aluno.

\subsection{Tubo fechado com diferentes comprimentos fixos - frequência fora do modo normal}

A Figura 13 mostra os dados adquiridos para uma frequência de excitação de $f_{e}=3300 \mathrm{~Hz}$, utilizando comprimentos do tubo de $L_{1}=16,0 \mathrm{~cm}, L_{2}=18,0$ $\mathrm{cm}, L_{3}=19,0 \mathrm{~cm}$ e $L_{4}=21,0 \mathrm{~cm}$. Com estes valores, só seria esperado que fosse obtida uma onda estacionária no primeiro e último caso, pois resultaria, a partir das equações (3) e $(5)$, que $n=m=$ 3 e $n=m=4$, respectivamente. No entanto, podemos ver pela Figura 13.a que ondas estacionárias foram formadas para todos os comprimentos. $\mathrm{Na}$ Figura 13.b comparamos as curvas teóricas com as obtidas e na Figura 13.c evidenciamos que todas as ondas estacionárias formadas possuem praticamente a mesma frequência, de $f_{o}=3300 \mathrm{~Hz}$.

A princípio, o aparecimento de uma onda estacionária, nesse caso, violaria a equação (3) pois, como dissemos, representaria um valor não inteiro para $n$. Entretanto, podemos ver que uma onda é formada independentemente do tamanho do tubo fechado e com praticamente a mesma frequência $(f=3300 \mathrm{~Hz})$. Porém, devemos notar que o perfil da onda formada dentro do tubo para comprimentos que não fornecem um número inteiro para $n$ na equação (3), não representa o esperado para um tubo fechado em suas extremidades - condição de máximos de pressão. Isso pode ser visto através da análise do perfil inicial dessas ondas na extremidade que contém o alto-falante (Figura 13.a, em $l=0,0$ $\mathrm{cm}$ ), em que para os casos com $L_{2}=18 \mathrm{~cm}$ e $L_{3}=$ $19 \mathrm{~cm}$ não se obtém um máximo. Neste caso, mesmo a extremidade estando fechada pelo próprio altofalante, ela não se comporta como uma extremidade fechada. O perfil da onda estacionária para um tubo fechado é alcançado somente quando $L_{1}=16,0 \mathrm{~cm}$, que representa $n=m=3$, e $L_{4}=21 \mathrm{~cm}$, que representa $n=m=4$.

As curvas obtidas podem ser melhor interpretadas se invertemos o eixo da abcissa e deslocamos o que seriam os finais das curvas do item a para que se encontrem em $l=0,0 \mathrm{~cm}$, Figura 13.b. A curva teórica para $L_{4}=21 \mathrm{~cm}$ mostra que o padrão obtido corresponde ao esperado, com máximos de amplitude encontrado em posições compatíveis com 


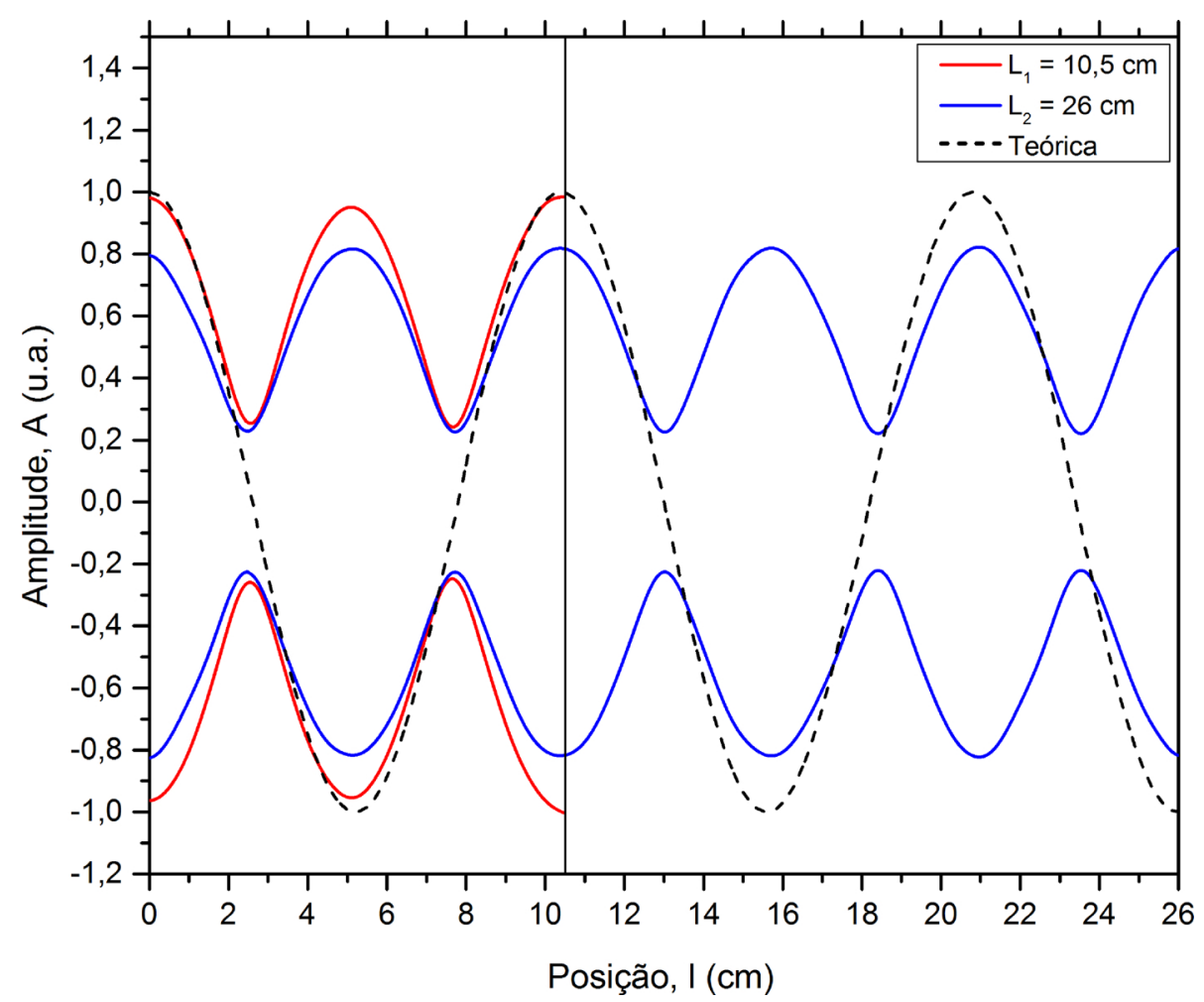

Figura 12: Envelope do sinal adquirido para o tubo fechado excitado com uma frequência de $f_{e}=3300 \mathrm{~Hz}$, para dois comprimentos distintos $L_{1}=10,5 \mathrm{~cm}(n=m=2)$ e $L_{2}=26,0 \mathrm{~cm}(n=m=5)$. O alto-falante se encontra à esquerda, posição $l=0 \mathrm{~cm}$. Para ambas as medidas, a amplitude da onda sonora aplicada foi a mesma.

a frequência utilizada. Podemos, entretanto, notar que as amplitudes serão menores para comprimentos que fornecem os valores mais distantes de $n$ inteiros. Outro fato que devemos estar atentos é a pequena variação no comprimento de onda que resulta do perfil de onda gerado pelo alto-falante não ser ideal, ou seja, há uma faixa de frequências próximas a de excitação que pode contribuir bastante com a formação de ondas estacionárias quando o comprimento está próximo de fornecer um número $n$ inteiro. No nosso caso essa faixa de frequências é da ordem de $250 \mathrm{~Hz}$, conforme pode ser evidenciado pela Figura 13.c. Esse efeito foi observado com o comprimento de $L=16,0 \mathrm{~cm}$, em que o valor de $L=15,75 \mathrm{~cm}$ resultaria em um $n$ inteiro. Nesse caso, a frequência encontrada está ligeiramente deslocada do valor utilizado na excitação, mas ainda dentro da faixa de $250 \mathrm{~Hz}$ mencionada. Para ilustrar isso, a curva teórica para $L_{1}=16 \mathrm{~cm}$ mostrada na Figura 13.b foi gerada utilizando a frequência de $f_{e}=3150 \mathrm{~Hz}$.

Assim, em um sistema não ideal, podemos afirmar que sempre haverá uma onda estacionária dentro do tubo, independente do comprimento utilizado, devido à natureza dos componentes do sistema, es- pecialmente o alto-falante. Enfatizamos que isso não invalida as equações utilizadas, apenas mostra que somente a frequência compatível com tal comprimento - condição de ressonância - contribuirá coerentemente naquele momento. Essa discussão será retomada com mais detalhes na última parte dos resultados deste artigo, quando apresentamos os resultados do experimento variando o comprimento.

\subsubsection{Tubo semiaberto com comprimento fixo e distância do alto-falante variada}

Com o objetivo de evidenciar os efeitos da distância sobre a forma de onda estacionária, realizamos este experimento com diferentes distâncias do alto-falante em relação à extremidade do tubo. Podemos observar, na Figura 14, que conforme a distância entre o alto-falante e a extremidade do tubo aumenta, o mínimo (ou nó) da onda estacionária se aproxima da extremidade, gerando também pequenos deslocamentos ao longo de toda onda. Ou seja, quanto maior a distância entre o alto-falante e a extremidade do tubo, mais próximo do esperado fica o perfil da onda no interior do tubo, com um mínimo 

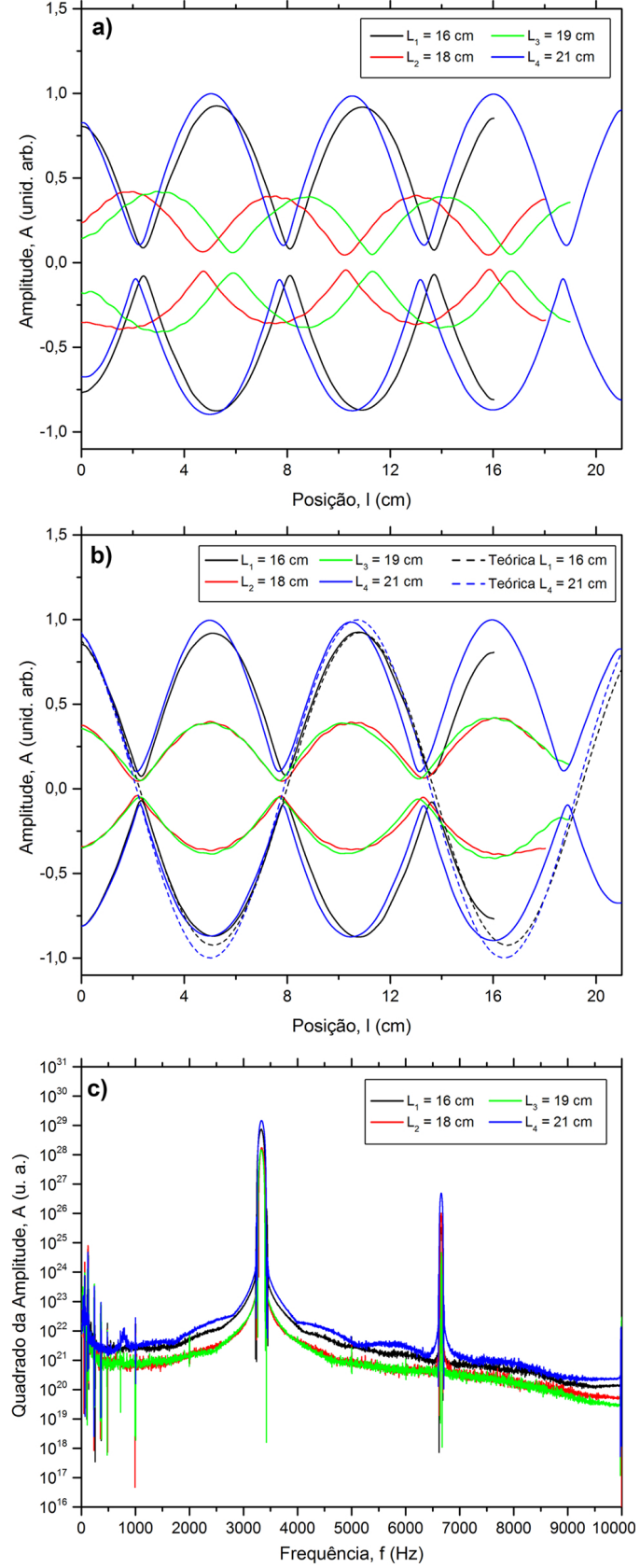

Figura 13: Sinais adquiridos para uma frequência de excitação de $f_{e}=3300 \mathrm{~Hz}$ e comprimentos $L$ do tubo diferentes. a) Envelope dos sinais originais para cada comprimento $L$, sendo $L=21 \mathrm{~cm}$ o comprimento correspondente a $n=m=4$. b) Sinais espelhados na abcissa, e deslocados fazendo com que os finais das curvas do item a sejam coincidentes em $l=0,0 \mathrm{~cm}$ e comparados às curvas teóricas (tracejadas). A curva teórica para $L=21 \mathrm{~cm}$ foi determinada utilizando a frequência de excitação utilizada no experimento enquanto a curva teórica para $L=16 \mathrm{~cm}$, que não corresponde exatamente a um comprimento cujo $n$ resultante seria um inteiro foi simulada utilizando $f=3150$ $\mathrm{Hz}$ para ilustrar o efeito de uma excitação não ideal. c) Transformada de Fourier do sinal original, evidenciando que a frequência característica é praticamente a mesma para todos as curvas adquiridas.

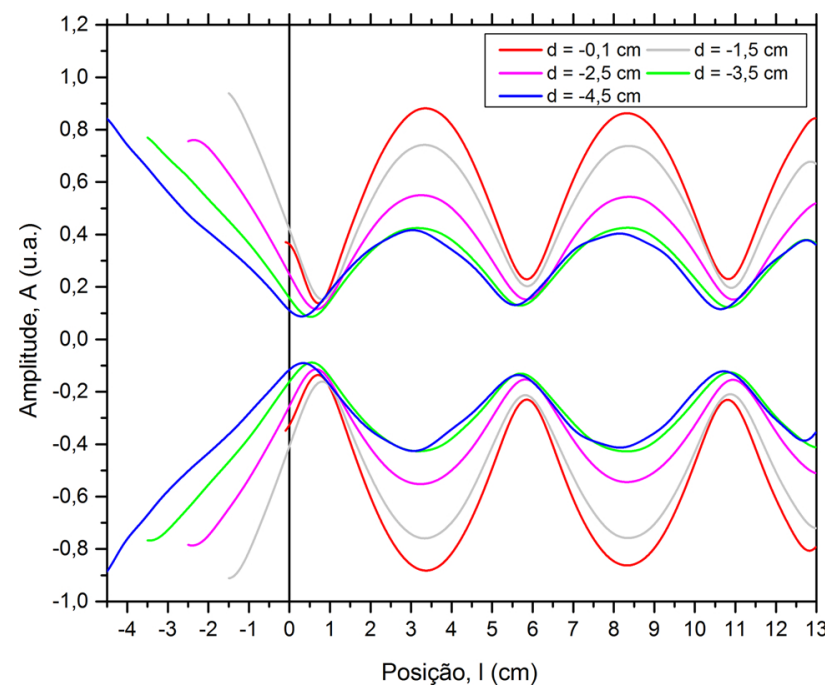

Figura 14: Envelope do sinal adquirido para uma frequência fixa, $f_{e}=3300 \mathrm{~Hz}$, em um tubo aberto em uma extremidade (esquerda) e com comprimento fixo, $L=13,0 \mathrm{~cm}$ ( $n=$ $3, m=2$ ), não levando em consideração a aproximação terminal. As distâncias negativas representam a distância do alto-falante em relação à extremidade do tubo (posição $l=0,0 \mathrm{~cm}$ ). Todas as medidas foram realizadas com a mesma amplitude na onda sonora aplicada. O microfone iniciou suas medidas da superfície do alto-falante, externo ao tubo.

de pressão exatamente na extremidade aberta do mesmo. Outro fato que pode ser evidenciado se refere à amplitude, sendo que quanto mais próximo o alto-falante está do tubo, maior a amplitude da onda estacionária formada dentro dele. Isso se deve às perdas para o ambiente externo quando a distância é maior. Ressaltamos apenas que não estamos levando em consideração a aproximação terminal, que para este caso não depende somente da relação com o diâmetro do tubo, mas da distância da fonte para com a extremidade aberta, espessura do tubo entre outros fatores que fazem o cálculo mais complexo [11]. Recomendamos a leitura da referência [11] para informações mais detalhadas dos parâmetros.

A influência da frequência da fonte sonora sobre o perfil da onda não foi estudada neste momento. Finalmente, é possível observar que mesmo com apenas $1 \mathrm{~mm}$ de espaçamento entre o alto-falante e a extremidade do tubo, apesar de não ser tão claro quanto para distâncias maiores como com $d=$ - 4,5 cm, o sistema pode ser considerado aberto. Isso também pode ser observado no experimento do tubo fechado, no qual, se o alto-falante não estiver realmente em contato com a borda do tubo, não permitirá que sejam visualizados com precisão os 
perfis de onda corretos. Este efeito foi observado durante os experimentos realizados nas montagens experimentais padrões (dados não mostrados).

\subsubsection{Tubo semiaberto com comprimento fixo e distância do alto-falante fixa}

Em um segundo caso, foram realizadas as medidas para duas frequências distintas, mantendo o alto-falante na posição $d=-4,5 \mathrm{~cm}$ em relação à extremidade do tubo. Essa distância foi escolhida por permitir uma boa representação do tubo semiaberto, baseada nos dados do experimento descritos no item anterior (4.2.1). Dados representativos desse experimento estão mostrados na Figura 15. Novamente, neste caso, não consideramos a aproximação terminal.

Podemos ver claramente pela Figura 15 que a frequência (número de nós) influencia a posição dos nós da onda estacionária dentro do tubo tanto quanto a distância do alto-falante em relação à extremidade do tubo. Para frequências mais altas, a tendência é que a onda observada represente mais fielmente a teoria, com mínimos de pressão exatamente na extremidade aberta, sem necessidade de se levar em conta outros fatores de ajuste. É importante salientar que os ruídos são atenuados para

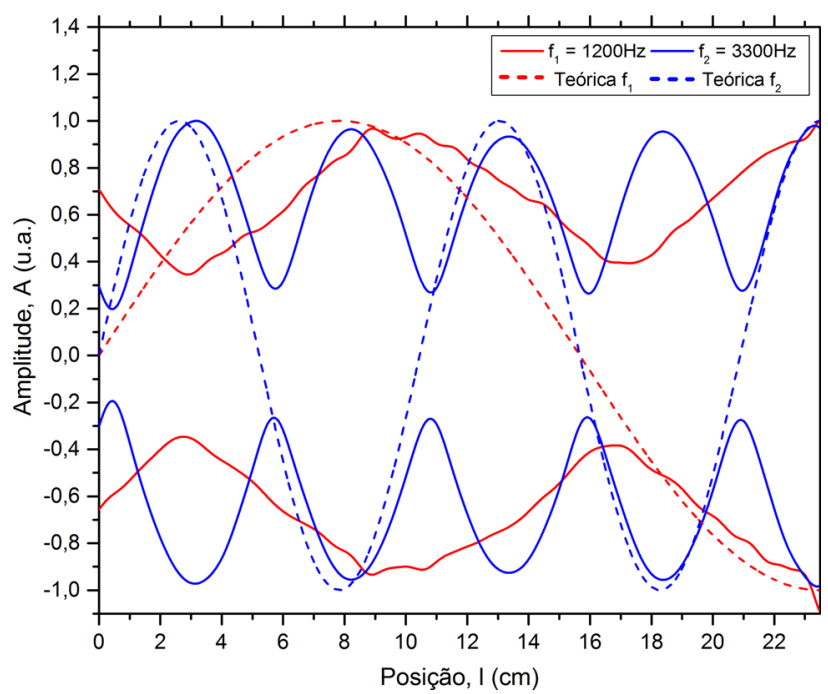

Figura 15: Envelope do sinal adquirido para duas frequências distintas, $f_{1}=1200 \mathrm{~Hz}(n=3, m=2) \mathrm{e}$ $f_{2}=3300 \mathrm{~Hz}(n=9, m=5)$, em um tubo semiaberto (aberto em uma extremidade) com comprimento fixo $L=$ $23,5 \mathrm{~cm}$ (não levando em consideração a aproximação terminal). A posição $l=0,0 \mathrm{~cm}$, indica a extremidade do tubo. $\mathrm{O}$ alto-falante foi posicionado a uma distância fixa $d=$ $4,5 \mathrm{~cm}$ da extremidade do tubo. frequências maiores, mesmo o sinal estando com uma amplitude menor, facilitando a aquisição do sinal e sua interpretação.

Neste experimento notamos a importância de se levar em consideração a aproximação terminal principalmente para frequências mais baixas ( $n$ menor). Porém, tal experimento foi limitado a apenas a observação do sinal sem a consideração de todos os parâmetros necessários para o correto cálculo da aproximação terminal [11]. Note também que a aproximação terminal prevê um afastamento do nó para o lado externo, o que não ocorreu neste caso. Esse efeito pode ser explicado pela frequência ligeiramente fora da ressonância se levado em consideração a aproximação terminal, o que seria similar ao mostrado pelo experimento do item 4.1.2. É importante ressaltar que a montagem experimental proposta nesse trabalho deve permitir um estudo detalhado acerca desse tema, o que se inclui, no momento, como perspectiva futura de aplicação deste sistema.

\subsection{Tubo fechado com comprimento variando}

Neste experimento, presente em quase todas as apostilas de laboratórios de física e equipamentos comerciais, há vários aspectos que podem dar origem a erros de interpretação. O primeiro deles está relacionado ao surgimento do primeiro pico de máximo, conforme mostra a Figura 16. É importante lembrar que, nesse caso, o tamanho útil (espaço entre o êmbolo e a extremidade onde existe o alto-falante) do tubo está variando e com ele a posição do microfone.

Com o tubo fechado, esperar-se-ia ver máximos na extremidade esquerda (indicados pelas setas), porém, neste experimento, a posição inicial corresponde ao comprimento $L=0,0 \mathrm{~cm}$ do tubo, sendo assim, menor que o tamanho mínimo para qualquer frequência diferente de zero $(\mathrm{n}<1$, na equação $(2))$, algo matematicamente incoerente. Porém, devido à proximidade do microfone ao alto-falante e somado ao tamanho reduzido do tubo $(L=0,0 \mathrm{~cm})$, observa-se que a amplitude do sinal recebido pelo microfone é grande, ocasionando o que parece ser um máximo, mas que na realidade não é. Veja que o envelope do sinal da frequência $f_{2}=3300 \mathrm{~Hz}$, linha vermelha da Figura 16, aparenta ser coerente com a teoria (formação de uma onda estacionária do tipo cossenoide), enquanto que para frequências mais baixas (linha preta, indicando uma frequência 


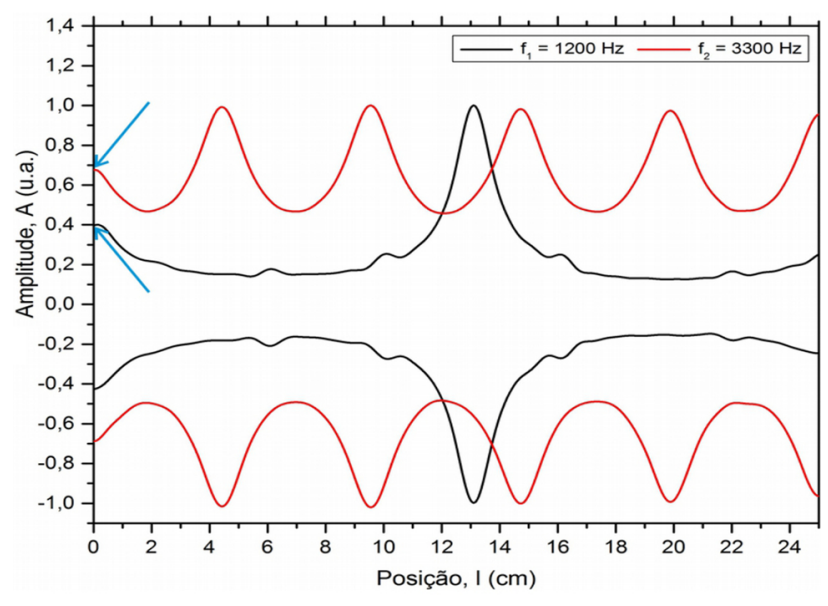

Figura 16: Envelope do sinal adquirido para o tubo fechado variando o comprimento para duas frequências distintas $f_{1}=1200 \mathrm{~Hz}$ e $f_{2}=3300 \mathrm{~Hz}$. O microfone anda em conjunto com a parede do êmbolo $L=l$. Neste caso o perfil visualizado não representa uma onda estacionária única, mas sim o perfil de ressonância do tubo. Note também que não existe máximo em $L=0,0 \mathrm{~cm}$, indicado pelas setas, a amplitude maior é devida a proximidade com o alto-falante, portanto, não condizente com o perfil de um tubo fechado também.

de $f_{1}=1200 \mathrm{~Hz}$,) a discrepância pode se tornar mais evidente.

Outra questão importante é quanto à forma de onda visualizada. Pode-se pensar que ao se variar o comprimento o tubo, o que o aluno faz é "varrer" o perfil de uma única onda estacionária. Porém, como foi mostrado anteriormente no experimento do item 4.1.2, para cada comprimento do tubo, uma onda estacionária diferente é observada. Assim, para uma dada frequência de excitação prática existem sempre modos normais independente do comprimento do tubo. O tubo funciona, então, como um amplificador, fazendo com que a onda estacionária ressoe com frequência próxima àquela inserida, compatível com o comprimento do tubo naquele momento, mas com amplitude mais baixa. É importante observar que o sinal referente à frequência $f_{2}=3300 \mathrm{~Hz}$ se assemelha ao perfil senoidal, porém, fica evidente com a frequência $f_{1}=1200 \mathrm{~Hz}$ que este não é senoidal, mas sim um perfil análogo ao pico de ressonância de um oscilador amortecido com a posição representando o eixo da frequência da fonte externa, o que está em bom acordo com a nossa interpretação para este efeito.

Após a realização de todos estes experimentos, uma questão pode ser colocada: a extremidade do tubo com o alto-falante é considerada aberta ou fechada? À primeira vista, a extremidade do tubo que contém o alto-falante poderia ser considerada aberta, afinal é a vibração da parede (cone do altofalante) que faz com que as moléculas de ar vibrem, dando origem às ondas estacionárias. Porém, caso o alto-falante esteja bem vedado na extremidade do tubo, como no experimento 4.1.1, nossos dados mostram que ele se comporta como um tubo fechado (claramente não o impedindo de atuar como oscilador). Isso ocorre porque quando temos um modo normal, com a formação da onda estacionária, além da superposição das ondas ocorre também o fenômeno de ressonância. A ressonância se dá pelo aumento da amplitude de oscilação devido a soma (em fase) das ondas geradas pela fonte sonora externa. Deste modo, isto faz com que a amplitude da onda (amplitude do deslocamento das moléculas de ar) seja maior que a própria amplitude de deslocamento do cone do alto-falante. Desta maneira, o valor da amplitude de vibração do alto-falante pode ser considerado desprezível, tornando-o assim uma parede rígida constituindo, então, o tubo fechado. É possível, ainda, aplicar este mesmo argumento para o experimento da corda vibrante. A fonte excitadora da corda (um pistão) pode ser comparada com o alto-falante para a onda sonora. Observa-se o mesmo fenômeno, neste caso, se a amplitude da fonte de vibração da corda for suficientemente pequena. Naquele ponto, é como se a corda estivesse presa. Se aumentamos a amplitude, podemos ter o deslocamento do ponto de mínimo de amplitude da corda.

Finalmente, devemos averiguar se o microfone de eletreto está medindo a pressão ou o deslocamento do gás no interior do tubo. Para isto, basta olharmos para as curvas obtidas com os experimentos do tubo fechado, por exemplo. Para o tubo fechado, as extremidades possuem máximos no sinal adquirido, devendo estes serem a representação dos máximos de pressão, já que o deslocamento é restrito pelas extremidades fechadas.

\section{Conclusões}

A montagem experimental utilizada para realizar o estudo sobre ondas estacionárias em um tubo mostrou ser eficiente na aquisição dos sinais, permitindo realizar medidas com maior precisão e introduzindo novas formas de visualização dos dados, facilitando o entendimento das configurações do tubo (fechado ou semiaberto). Como proposta para o uso em um 
laboratório de ensino de física, recomendamos o uso do sistema fechado, realizando a vedação das extremidades. Os resultados obtidos mostraram que este procedimento evita o aparecimento de efeitos não descritos na teoria básica, como a formação deslocada dos nós nos tubos abertos, além da própria interferência entre os diferentes equipamentos que estejam sendo utilizados simultaneamente ou a confusão entre os perfis de tubos abertos e fechados.

Outra questão importante se refere à não utilização da variação do comprimento do tubo em conjunto com o microfone. Alterar o tamanho do tubo em conjunto com a posição da medida pode trazer complicações e dificuldades na interpretação dos resultados que se não forem muito bem discutidas poderão levar o estudante ao erro e até mesmo a um aprendizado errado. Desta forma, acreditamos que deva ser mantido apenas o microfone em movimento, mantendo o comprimento do tubo fixo. Isso pode ser facilmente realizado com o uso de uma vareta interna ao braço do êmbolo passando pelo seu centro.

Como forma de aprimorar as medidas, a utilização de frequências acima de $3300 \mathrm{~Hz}$ em substituição às frequências tipicamente adotadas em torno de 1000 $\mathrm{Hz}$, faz com que a forma de onda observada seja mais pura, reduzindo interferências e fazendo com que o som seja menos audível devido a atenuação pelo material do tubo (vidro). Isto também permite a utilização de tubos de comprimentos menores, pois frequências maiores representam comprimentos de onda menores. Desta forma, tubos com $30 \mathrm{~cm}$ seriam o suficiente em substituição aos tubos de $1 \mathrm{~m}$ usuais nestas montagens. Isso reduziria o tamanho do aparato para $1 / 3$ do valor atual, reduzindo também os custos de construção e de armazenamento.

Incentivamos que os alunos sempre verifiquem seus resultados e caso não sejam compatíveis como o descrito pela teoria, verifiquem as possíveis fontes de erro. Esperamos ter mostrado, através deste trabalho, que o estabelecimento de novas formas de realizar medidas pode facilitar a realização das mesmas, e, principalmente, evitar que interpretações errôneas de conceitos físicos possam ser geradas a partir desses experimentos.

\section{Sugestões}

Uma sugestão para futuros trabalhos e implementações do sistema descrito nesse artigo é a utilização da saída de áudio do próprio Arduino para geração de ondas de excitação. Isso facilitaria o controle do sistema, além de possibilitar a redução de mais dois componentes do sistema, como o gerador de onda e o frequencímetro. Outra vantagem seria a possibilidade de gravação da onda inserida para comparações futuras. O sistema de movimentação do êmbolo e microfone pode ser realizado por um sistema de correias ou cabos, o que facilita o tracionamento dos objetos em comparação com o utilizado em forma de cremalheira. Por fim, poder-se-ia utilizar o tubo com as duas extremidades abertas, algo que dificilmente é tratado nos experimentos, livros e apostilas de laboratório. Para este caso, a dificuldade estaria em se modificar o tamanho do tubo, pois teria de se usar o tubo por completo, necessitando do uso de vários tamanhos de tubos diferentes se este fosse o caso.

\section{Agradecimentos}

Aos técnicos dos laboratórios de ensino Antenor F. Petrilli Filho, Cláudio B. Bretas, Jae A. de Castro Filho, Marcos J. Semenzato. Aos técnicos da oficina mecânica Ademir Morais, Carlos A. Pereira, Ricardo H. Rodrigues. Ao professor Francisco G. Guimarães pelas discussões acaloradas e sugestões iniciais. Ao professor Alberto Tannús do Centro de Imagens In Vivo por Ressonância Magnética, pelo empréstimo de materiais necessários às medidas e ao técnico João M. P. Nogueira da Oficina Eletrônica do Grupo de Óptica. À Geisiane R. da Silva pelas revisões e sugestões. Ao Programa Unificado de Bolsas de Estudo para Estudantes de Graduação da Universidade de São Paulo, ao Instituto de Física de São Carlos e a Universidade de São Paulo.

\section{Referências}

[1] S.C. SAAB, Fábio Augusto Meira Cássaro e André Maurício Brinatti, Caderno Brasileiro de Ensino de Física 22, 112 (2005).

[2] L.P. Vieira, D.F. Amara e V.O.M. Lara, Revista Brasileira de Ensino Física 36, 1 (2014).

[3] F. Sears, Física II (Livros Técnicos e Científicos Editora LTDA, Rio de Janeiro, 1989), v. 2.

[4] A.P. French, Vibration and Waves: The M.I.T Introductory Physics Series (W.W. Norton \& Company, New York, 2003), 198 p.

[5] J.S.S. Vasconcelos, R.J.A. Vieira, N.S. Soeiro e G.S.V. Melo. in: I Workshop de Vibração Acústica da Região Norte, Tucurui, 2011. 
[6] D.A. Bohn, Jornal of the Audio Engineering Society 36, 223 (1988), disponível em http://www.pearl-hifi.com/06_Lit_ Archive/15_Mfrs_Publications/Rane_Notes/ Fundamentals/Enviromental_Effects_on_the_ Speed_of_Sound.pdf

[7] N.G.R.F. Melo, L.M. Silveira, M.V.G. de Morais, M.A.A. Nunes, M.H.A. Gomes e A.B.S. Oliveira in: VI Congresso Nacional De Engenharia Mecânica - CONEM, Campina Grande, 2010, disponível em https://fga.unb.br/articles/0000/ 5897/conen20110_tigad.pdf

[8] J. Nichols, Theoretical and Experimental Comparison of Pressure in a Standing Wave Tube with Attenuation (University of Illinois, Urbana-Champaign), disponível em https://courses.physics.illinois.edu/ phys406/NSF_REU_Reports/2011_reu/John_ Nichols/John_Nichols_Senior_Thesis.pdf.

[9] R.V. Salvo, M.T.F. Mairink, E.C. de Oliveira e E.B. Teodoro, in: $15^{\circ}$ Simpósio do Programa de Pós-Graduação em Engenharia Mecânica - POSMEC, Uberlândia, 2005, disponível em http://www.posgrad.mecanica.ufu. br/posmec/15/pdf/POSMEC056.pdf

[10] S. Errede, Mathematical Musical Physics of the Wave Equation - Part 2 (University of Illinois, Urbana-Champaign, 2015), disponível em https://courses.physics.illinois.edu/ phys406/Lecture_Notes/P406P0M_Lecture_ Notes/Mathematical_Musical_Physics_of_The_ Wave_Equation_Part2.pdf

[11] J. Liljencrants, End Correction at a Flue Pipe Mouth, disponível em http://www.fonema. se/mouthcorr/mouthcorr.htm acessado em $12 / 8 / 2016$.

[12] M.A.B. Freitas, Medindo a Velocidade do Som com o Microfone do PC. Tese de Conclusão de Curso, Instituto de Física, Universidade Federal do Rio de Janeiro, Rio de Janeiro, 2005. 